\title{
ELIMU-MDX: a web-based, open-sourrce platform for storage, management and analysis of diagnostic qPCR data
}

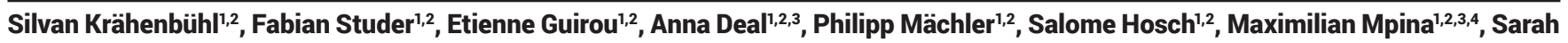
Mswata $^{4}$, Claudia Daubenberger ${ }^{1,2}$ \& Tobias Schindler ${ }^{*, 1,2}$

\section{ABSTRACT}

The Electronic Laboratory Information and Management Utensil for Molecular Diagnostics (ELIMU-MDx) is a user-friendly platform designed and built to accelerate the turnaround time of diagnostic qPCR assays. ELIMU-MDx is compliant with the MIQE guidelines and has extensive data-import capabilities for all major qPCR instruments by using the RDML data standard. This platform was designed as an open-source software tool and can be accessed through the web browser on all major operating systems.

\section{METHOD SUMMARY}

ELIMU-MDx is an open-source web-application developed using PHP to analyze, manage, validate and store user-provided qPCR data in a MySQL database.

\section{KEYWORDS}

diagnostic $\cdot$ ELIMU-MDx $\cdot$ infectious diseases $\cdot \mathrm{MIQE} \cdot \mathrm{qPCR} \cdot \mathrm{RDML}$

${ }^{1}$ Department of Medical Parasitology \& Infection Biology, Swiss Tropical \& Public Health Institute, Basel, Switzerland; ${ }^{2}$ University of Basel, Basel, Switzerland; ${ }^{3}$ Equatorial Guinea Malaria Vaccine Initiative, Malabo, Republic of Equatorial Guinea; ${ }^{4}$ Bagamoyo Research \& Training Centre, Ifakara Health Institute, Bagamoyo, United Republic of Tanzania; *Author for correspondence: tobias.schindler@unibas.ch

BioTechniques 68: 22-27 (January 2020) 10.2144/btn-2019-0064
The global market of in vitro diagnostics, comprising tools to detect, diagnose or monitor diseases, is estimated to be worth US\$40-45 billion [1]. One of the fastest growing areas is the identification of infectious diseases using molecular diagnostics, which is becoming an integral part of medical practice and public health worldwide [2]. Molecular diagnostic tools, especially nucleic acid amplification techniques, provide faster, more sensitive and often more cost-effective diagnoses than traditional culture methods for microbiological analysis [3]. Quantitative PCR (qPCR) is a well-established method for the detection, quantification and typing of bacteria, viruses, fungi and protozoa in the areas of clinical and veterinary diagnostics, as well as food safety [4]. Among the biggest advantages of qPCR-based diagnosis are the universality in designing and developing new assays as well as the widespread availability of the technology. Moreover, qPCR also allows quantification and genotyping of pathogens. With the rapid spread of qPCR into routine diagnostics, standardization and validation processes of qPCR assays and data had to be addressed [4]. A first step was the publication of the MIQE guidelines [5], which has led to a certain degree of standardization in the validation process of qPCR assays across different laboratories. In order to standardize the qPCR data format the real-time $P C R$ data markup language (RDML) [13], which stores the data in a compressed extensible markup language file, was designed [6]. RDML stores the raw data acquired by the qPCR instrument as well as the information required for its interpretation, such as sample annotation and cycling protocol. A comprehensive review of 27 open-access software packages and tools for qPCR data analysis reported that most tools use their own file format and only a fraction of the currently existing tools support the standardized data exchange format RDML [7]. The lack of a common standard for qPCR data makes it difficult to share and analyze data across different qPCR platforms.

The high-throughput character of qPCR analysis in infectious disease diagnosis easily leads to a rapid accumulation of raw data that needs to be quality controlled and interpreted in order to correctly identify and quantify infectious agents. Therefore, access to well-designed, user-friendly and semi-automated software facilitating the storage, quality control (QC) and analysis of diagnostic qPCR data is needed.

Controlled human malaria infection (CHMI), the deliberate exposure of human volunteers to fully infectious malaria parasites, has proven to be safe and well tolerated, and accelerates the evaluation of malaria vaccines candidates in clinical trials $[8,9]$. Volunteers participating in CHMls must be monitored closely to ensure volunteer safety. Blood is drawn twice daily for up to 2 weeks and screened for Plasmodium falciparum parasites by thick blood smear microscopy, and after the first appearance of blood-stage parasites the antimalarial treatment is initiated. Nowadays, more sensitive qPCR is increasingly used in CHMI to closely monitor parasitemia levels, which improves the safety of participants [10]. In order to manage the vast amount of qPCR data generated during a series of $\mathrm{CHMI}$ studies with more than 250 individuals included and conducted in Tanzania and Equatorial Guinea, we decided to design and build a platform that facilities the analysis and interpretation of diagnostic qPCR data. We identified several key features which in our opinion would be required for such software: - Highly adaptable and integrative: a so- 
lution that can be easily adapted to a variety of qPCR assays, supports semiautomated data analysis and integrates into existing sample workflow;

- Data traceability throughout analysis process: the use of an audit trail and community data standards to ensure high data integrity;

- Accessibility and reliability of software: Platform-independent, open-source and low-maintenance software that could be deployed rapidly to new laboratories and other projects using qPCR analysis as a monitoring tool.

To address these needs, we developed the web-based, open-source platform ELIMU-MDx (Electronic Laboratory Information and Management Utensil for Molecular Diagnostics).

\section{MATERIAL \& METHODS \\ ELIMU-MDx development}

ELIMU-MDx combines a relational SQL database with a user-friendly web interface programmed in PHP 7.2 using the framework Laravel 5.8 and the tool Laravel Nova 2.0. The platform is accessed through a web browser (Google Chrome, Firefox and Safari were successfully tested) and needs to be hosted on a nginx or apache server. It validates user-provided data and stores it in a MySQL 5.7 database, and where appropriate constraints ensure data consistency.

\section{Analysis of qPCR data by ELIMU-MDx}

qPCR data generated for diagnostic purposes have different requirements to software than data generated for other qPCR applications. Most importantly, the consistency and reliability of the data needs to be ensured. Each qPCR run needs to have controls in place to test for contamination (preventing false-positive results) and to test the performance of extraction and qPCR reaction (preventing false-negative results). All data uploaded to ELIMU-MDx is quality controlled based on several controls and predefined criteria. Only samples that pass all QC thresholds are considered for further analysis. Additionally, a sample measured multiple times with the same assay is automatically considered as technical replicates, even if the data are derived from different experiments. This allows users to repeat sample analysis if needed.
ELIMU-MDx processes qPCR data derived from both major qPCR detection technologies. Assays using dye-based (e.g., SYBR or Eva-Green dyes) or TaqMan-based detection can be analyzed. The quantification cycle (Cq) values, the number of cycles needed to reach a fluorescence threshold, is extracted from the RDML file and used for qualitative and quantitative interpretation of the qPCR assay. In case of a dye-based detection, only $\mathrm{Cq}$ values from samples whose amplicon has a melting temperature within a predefined range are considered for further analysis. Since the melting temperatures are not included in the RDML version 1.1 from Bio-Rad (CA, USA), CFX Maestro (Version 4.1.2433.1219) and RDML version 1.2 from MyGo Pro PCR Software (Version 3.4; IT-IS Life Science, Republic of Ireland), they need to be uploaded separately in addition to the RDML file.

The current version of ELIMU-MDx supports absolute quantification of the qPCR target molecules using the slope and intercept derived from the linear relationship between $\mathrm{Cq}$ values and serially diluted standards of known nucleic acid concentrations.

The assay definition file (ADF) includes all assay-specific parameters that together define the assay. ADF is used by ELIMU-MDx for data analysis using different thresholds and parameters depending on the actual experimental settings. The advantage of defining all assay parameters in one single file is its universality and easy adaption to new qPCR assays. An example for an ADF is provided as an excel file (Supplementary file 1).

\section{RESULTS \& DISCUSSION}

\section{ELIMU-MDx overview}

The structure of ELIMU-MDx is shown in Figure 1. ELIMU-MDx consists of three modules - the sample module, the assay module and the data module - which are connected by an experiment. Each of the modules is supplied with data and parameters from predefined components. These components contain sample-, assay- or data-specific information that are necessary to interpret and document the experiments. The ADF, which contains all parameters necessary to analyze samples measured with the appropriate qPCR assay, is linked through the assay to the experiment. The experiment is considered the central node of ELIMU-MDx and combines the sample and assay metadata with qPCR raw data. Upload of standardized data is followed by initial QC and automated analysis and eventually results in a downloadable result file, which can be used for generating reports or statistical computing and data visualization by any statistical software.

Examples of the ELIMU-MDx user interface are illustrated in Figure 2. All features are accessible through a sidebar (Figure 2A). The oligo details are shown as an example for the level of detail that is provided by ELIMU-MDx's virtual freezer (Figure 2B). As part of the built-in sample inventory, the storage position of each sample can be displayed (Figure 2C). Results are automatically displayed after uploading the qPCR RDML files and samples with inconclusive results can be easily identified using the custom-build filters (Figure 2D). The integrated audit trail records all major events and therefore ensure high data integrity (Figure 2E).

\section{ELIMU-MDx is built as an open-source web-app}

ELIMU-MDx is a user-friendly platform designed and built to accelerate the turnaround time of diagnostic qPCR assays. The advantages of web-based applications include rapid deployment, cross-platform compatibility and easy application development [11]. A detailed guide to setup the platform, with step-by-step instructions, is provided at https://sparclex.github.io/elimu. The low-maintenance structure is an additional advantage, particularly in resource-limited settings. The source code is open source and distributed under a MIT license and is publicly available for download. ELIMU-MDx is unlike most commercially available qPCR software in that it is not a black box. All features of ELIMU-MDx are completely disclosed and can be improved or changed by other members of the scientific community or even used separately in other software.

The ELIMU-MDx can be accessed through https://elimu.ch, where we have created a specific user account for demonstration purposes (login: demo.user@elimu. ch, password: elimu2019). 


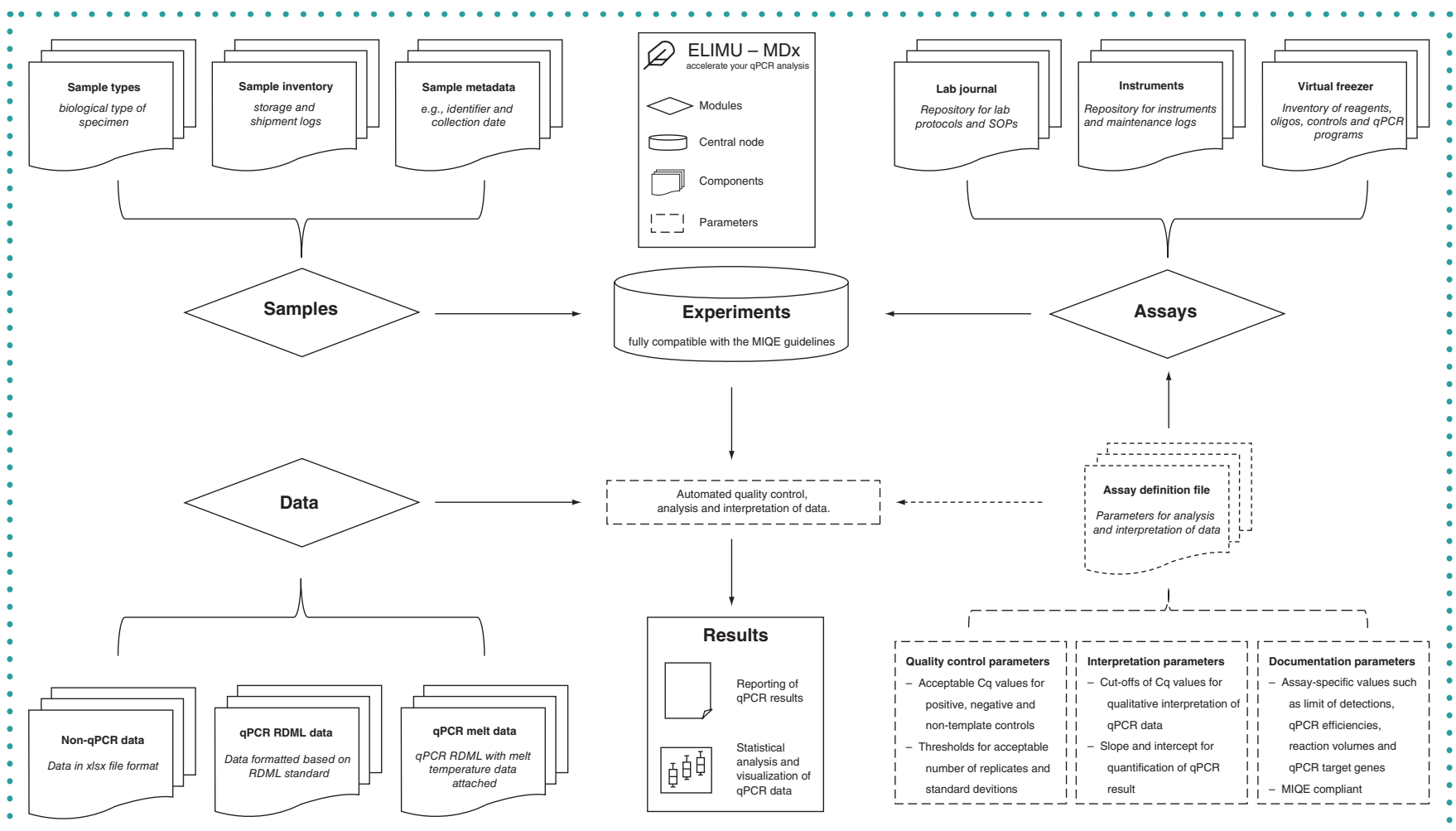

Figure 1. Structure of ELIMU-MDx. The sample, assay and data modules are connected to create an experiment, which is the central node of the platform. Assay-specific parameters are provided through the assay definition file.

Cq: Quantification cycle; RDML: Real-time PCR data markup language; SOP: Standard operating procedure.

\section{ELIMU-MDx uses the RDML data} standard, making it qPCR instrument independent

ELIMU-MDx supports RDML, a structured and universal data standard for exchanging qPCR data. RDML files contain all information to understand the experimental setup, reanalyze the data and interpret the results. The use of the RDML data standard allows using data from different qPCR instruments. The platform was successfully tested with RDML files version 1.1 and 1.2 from the Bio-Rad CFX96 Real-Time System and the MyGo Pro qPCR instrument.

\section{Semiautomated data QC \& analysis improves sample-to-result turnaround times}

The use of the standardized data format of RDML files allowed us to automate QC, analysis, and validation of qPCR data. Initial QC includes the automated analysis of the positive, negative and nontemplate control of each qPCR run uploaded based on provided cut-off values. The internal control of each sample is used to validate the extraction and amplification performance. Based on the $\mathrm{Cq}$ values provided by the
RDML file and a set of assay-specific parameters, as defined by the ADF, QC as well as qualitative and/or quantitative analysis is performed.

The ELIMU-MDx platform was extensively tested with diagnostic qPCR data generated during CHMI studies in Tanzania (total of 478 samples) and Equatorial Guinea (total of 2907 samples). To demonstrate the advantages and speed of ELIMUMDx, we used three separate qPCR runs generated during $\mathrm{CHMI}$ studies for a headto-head comparison with data-management solutions based on Microsoft (MS; WA, USA) Excel and a previously used custom-built MS Access database (Figure 3). At each of the four main steps of qPCR data management, the use of ELIMU-MDx allows for faster processing of qPCR data. With ELIMU-MDx the initial QC of the qPCR run is automatically performed during the upload of the qPCR data and by using a single, standardized RDML file containing the qPCR raw data, the upload procedure to ELIMU-MDx is simple and faster. Once the data are uploaded, the Cq values are interpreted and the result is linked to the sample. At this step, after a manual review of the data, the results are summarized and ready to be reported. Overall, the entire process from initial QC to a final report took approximately 15 min using ELIMU-MDx. This is a significant reduction in time spent on QC and analysis of the QPCR data when compared with the two other data analysis tools.

\section{ELIMU-MDX simplifies documentation,} statistical analysis \& visualization of qPCR experiments

The combination of assay-specific information, as provided by the ADF, sample metadata and data provided by the RDML file, contains all information required by the MIQE guidelines. Table 1 lists all information documented for each qPCR experiment as defined by the ELIMU-MDx platform.

The ELIMU-MDx platform also serves as a laboratory information and management system. We added general features of a laboratory information and management system to the ELIMU-MDx platform. A unique storage position can be assigned to each sample to keep track of sample storage. If samples are shipped, all details of the shipment will be logged. Apart from qPCR data, all other types of results can 


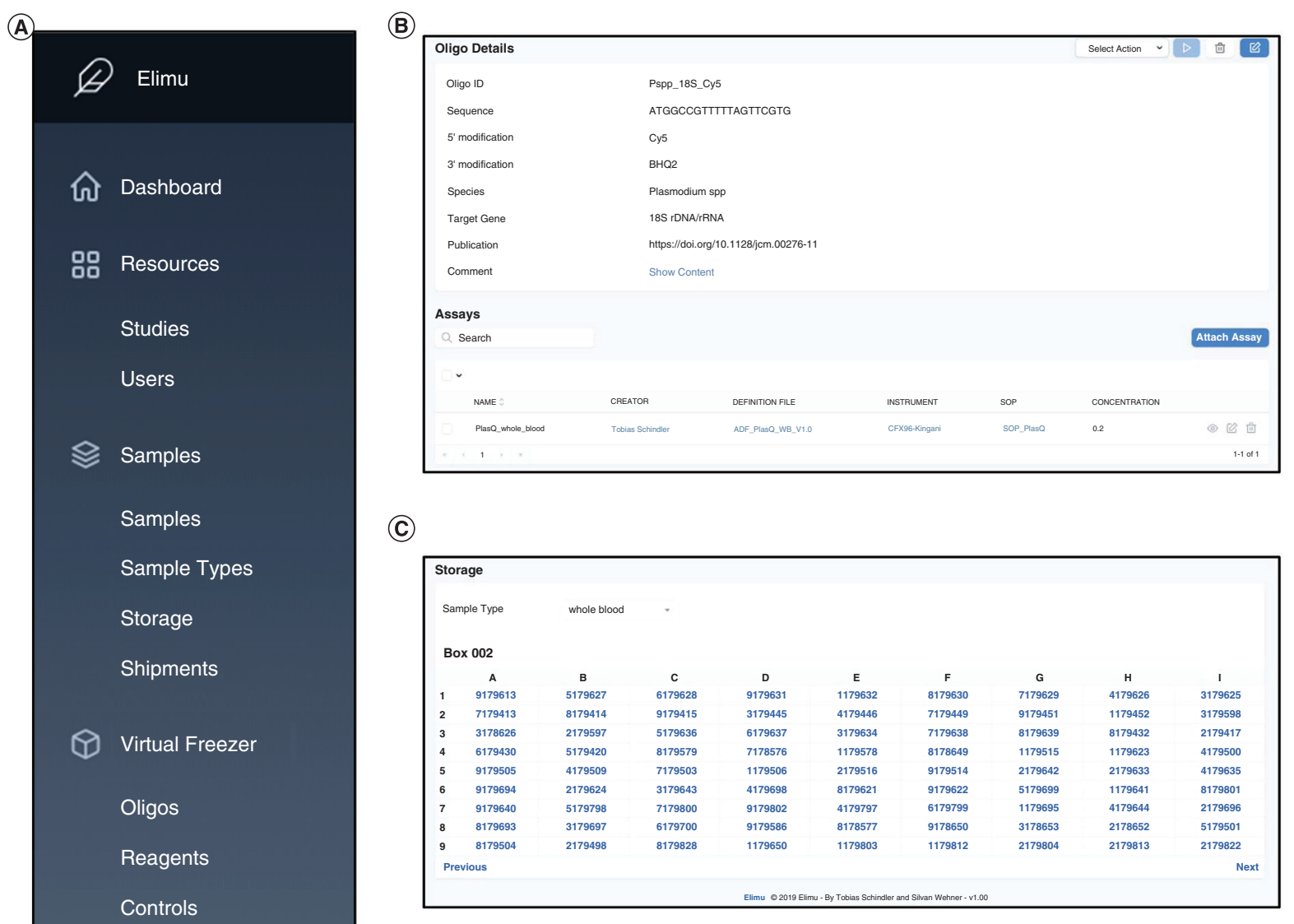

(D)

$\downarrow$ Analysis

Experiments

Assays

Definition Files

$\ominus$ Results

Results

Lab Journal

SOPs

Lab QC

Instruments

Maintenances

Audit

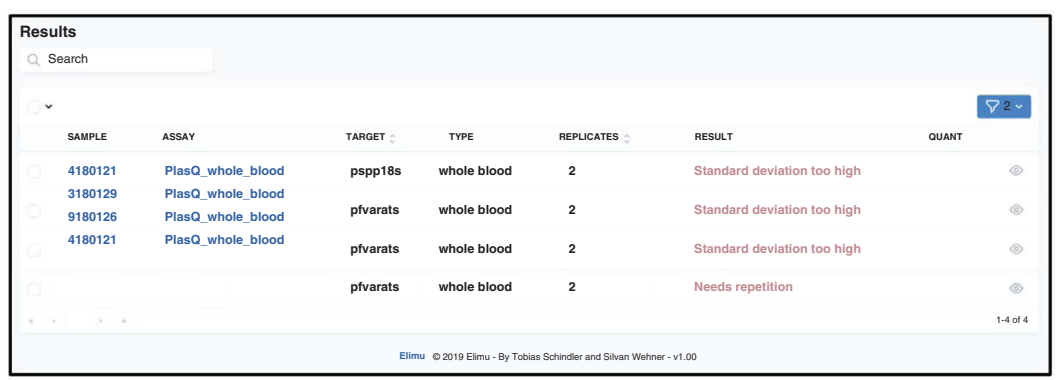

(E)

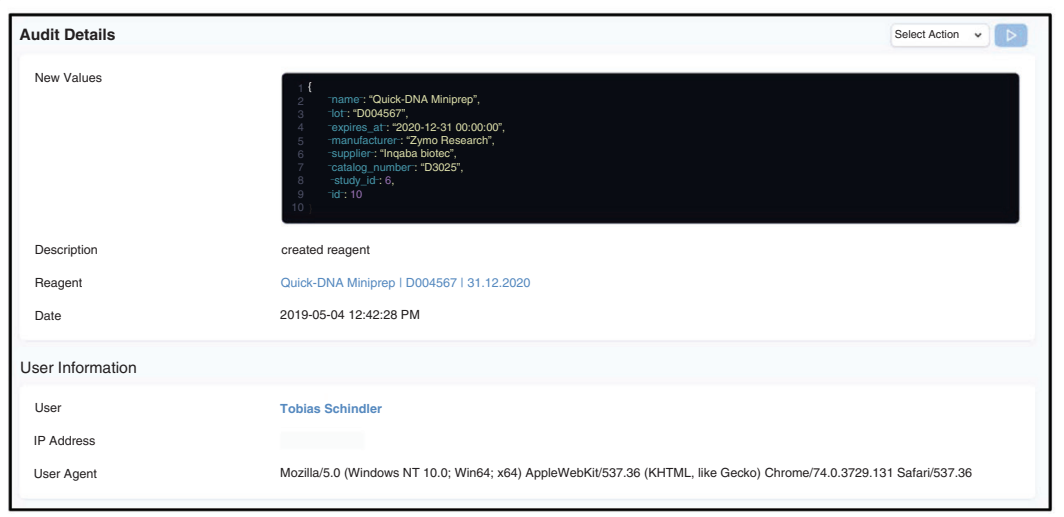

Figure 2. User interface of ELIMU-MDX. (A) Side bar for navigation within ELIMU-MDx. (B) Oligo details as an example for an ELIMU-MDx component. (C) Automatically assigned storage position for samples. (D) Samples that need special attention can be filtered in the results section. (E) ELIMU-MDx audit trail. Cq: Quantification cycle; SOP: Standard operating procedure. 
Table 1. Information documented for each experiment and sample.

\begin{tabular}{|c|c|c|}
\hline Modules & Component & Description \\
\hline Sample module & Sample metadata & $\begin{array}{l}\text { Required is a unique identifier, collection date and biological type of sample. } \\
\text { Optional information such as demographics can be added }\end{array}$ \\
\hline \multirow[t]{2}{*}{ Assay module } & Lab journal & $\begin{array}{l}\text { Contains detailed information for the associated protocols and standard operating } \\
\text { procedures }\end{array}$ \\
\hline & Virtual freezer & $\begin{array}{l}\text { Contains information for: } \\
\text { Oligonucleotides: sequences, link to original publication } \\
\text { Reagents: qPCR reagents with lot number and expiry date and extraction kits for } \\
\text { nucleic acids } \\
\text { Controls: name and concentration of positive controls } \\
\text { qPCR programs: Cycling conditions and detection channels used. qPCR instrument } \\
\text { software name and version }\end{array}$ \\
\hline \multirow[t]{2}{*}{$\begin{array}{l}\text { Assay definition } \\
\text { file }\end{array}$} & Analysis parameters & $\begin{array}{l}\text { Assay-specific parameters that are used to analyze and interpret the qPCR data. For } \\
\text { details, refer to the ELIMU-MDx user guide }\end{array}$ \\
\hline & Documentation parameters & $\begin{array}{l}\text { Additional assay-specific parameters, e.g., qPCR efficiencies, which are used for } \\
\text { documentation purposes only }\end{array}$ \\
\hline
\end{tabular}

be uploaded and attached to the samples, and therefore linked with the qPCR result. Importantly, the structured data generated by ELIMU-MDx allows use of the R language and environment for statistical computing and visualization of qPCR data without extensive data cleansing.

\section{Integrity \& traceability of qPCR data in ELIMU-MDx}

All qPCR data uploaded to ELIMU-MDx are protected from accidental or intentional modifications. The original qPCR raw data, as a RDML file, are attached to the results. The Cq values cannot be changed or deleted; the only option is to exclude replicates. Exclusion of $\mathrm{Cq}$ values is visually indicated and can be reversed. Each modification of the data is attributed to a user. The use of personal accounts that are password protected and the automatic creation of an audit trail that records and logs all major changes are in line with US FDA regulations on electronic records and electronic signatures (FDA regulation 21 CFR Part 11).

qPCR is a powerful tool that is used in different areas of infectious disease diagnostics, and it has become a key technology for the detection of viruses, bacteria, fungi and parasites by increasingly replacing traditional diagnostic techniques [4]. For laboratories, especially

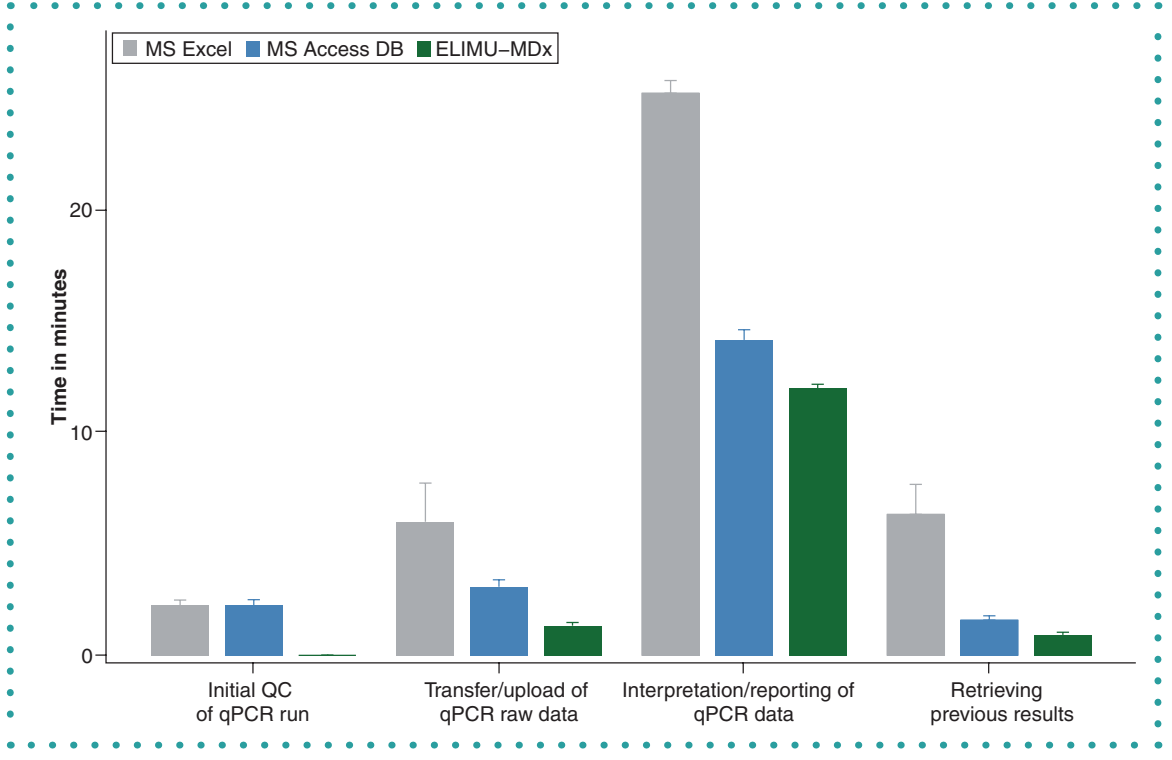

Figure 3. Analysis of qPCR data using ELIMU-MDx. Time spent on different steps of qPCR data management compared between Microsoft Excel, a custom-built Microsoft Access database and ELIMU-MDX.

QC: Quality control.

if they generate diagnostic data for clinical trials, the adequate storage, curation and analysis of qPCR data can be a challenge. Currently available software solutions, commercial or open source, are not well suited for the management and analysis of diagnostic qPCR data. Often a combination of several tools is used with an inadequate documentation of this essential step of the qPCR workflow.
We have built the ELIMU-MDx platform as a response to the large amount of qPCR data generated during clinical trials, including $\mathrm{CHMI}$ studies. Due to its integration into the sample workflow and automation of the qPCR QC and preliminary analysis, the use of ELIMU-MDx accelerates the sampleto-result turnaround time significantly. The use of the RDML standard allows analysis of the qPCR data in a streamlined and trans- 
parent way without losing the association between the final result (interpretation of the qPCR data) and the raw data (fluorescent data of the qPCR run). We also placed importance on the documentation of the qPCR experiments by attaching metadata from the samples and assays to the qPCR data. Maintaining an audit trail is a regulatory compliance requirement; therefore, we integrated an automated, time-stamped audit trail that allows the reconstruction of all events related to the creation, modification or deletion of electronic records.

ELIMU-MDx is open source, licensed under the permissive MIT license. Disclosing the software code used for data analysis should be the standard and will help to increase the reproducibility of data analysis [12]. Because ELIMU-MDx is an open-source project, contributions and feedback from the community are highly encouraged and will be an important factor in improving and adaptation of ELIMU-MDx over time.

\section{FUTURE PERSPECTIVE}

With the rapid spread of qPCR-based technologies for infectious disease diagnostics, the development and implementation of software for qPCR data analysis will continue to gain importance, particularly in the framework of clinical intervention studies that need to follow good laboratory practices. Open access software tools that enable reproducible data analysis, audit trails and long-term storage of raw and analyzed qPCR data are likely to become essential in experimental clinical research and diagnostics in the foreseeable future.

\section{AVAILABILITY \& REQUIREMENTS}

Software name: ELIMU-MDx

Software home page: https://www.elimu.ch Software source: https://github.com/ Sparclex/elimu
Open-source license: MIT license

Operating systems(s): OS independent, designed as a web-app

Supported browsers: Firefox, Google Chrome and Safari.

Programming languages: PHP

Other requirements: https://sparclex.github. io/elimu/dev-docs/requirements.html

Any restrictions to use by non-academics: no limitations

\section{AUTHOR CONTRIBUTIONS}

Concept and design: SK, FS, CD, TS. Programming: SK. Beta testing, troubleshooting and providing feedback: PM, EG, AD, SH, MM, SM, TS. Drafting the manuscript: SK, TS, CD. All authors read and approved the final manuscript.

\section{ACKNOWLEDGMENTS}

The authors would like to thank the Swiss Federal Office for Civilian Service for providing the possibility for SK, FS and PM to do their civilian service at the Swiss Tropical and Public Health Institute, and subsequently for making the development of ELIMU-MDx possible.

\section{FINANCIAL \& COMPETING INTERESTS DISCLOSURE}

The development of ELIMU-MDx was funded by the public-private partnership, the Equatorial Guinea Malaria Vaccine Initiative (EGMVI), supported by the Government of Equatorial Guinea, Marathon EG Production Limited, Noble Energy and Atlantic Methanol Production Company. The authors have no other relevant affiliations or financial involvement with any organization or entity with a financial interest in or financial conflict with the subject matter or materials discussed in the manuscript apart from those disclosed.

No writing assistance been used in the creation of this manuscript.

\section{SUPPLEMENTARY DATA}

To view the supplementary data that accompany this paper please visit the journal website at: www.future-science. com/doi/suppl/10.2144/btn-2019-0064

\section{DATA SHARING STATEMENT}

The source code of ELIMU-MDx is fully disclosed and available.

\section{OPEN ACCESS}

This work is licensed under the AttributionNonCommercial-NoDerivatives 4.0 Unported License. To view a copy of this license, visit http://creativecommons.org/licenses/ by-nc-nd/4.0/

\section{REFERENCES}

1. Morel C, McClure L, Edwards S et al. Overview of the diagnostics market. In: Ensuring Innovation in Diagnostics for Bacterial Infection: Implications for Policy. European Observatory on Health Systems and Policies (2016).

2. Patrinos GP, Danielson PB, Ansorge WJ. Molecular diagnostics. In: Molecular Diagnostics. Elsevier, 1-11 (2017).

3. Caliendo AM, Gilbert DN, Ginocchio CC et al. better tests, better care: improved diagnostics for infectious diseases. Clin. Infect. Dis. 57(Suppl. 3), S139-S170 (2013).

4. Kralik P, Ricchi M. A basic guide to real time PCR in microbial diagnostics: definitions, parameters, and everything. Front. Microbiol. 8, 108 (2017).

5. Bustin SA, Benes V, Garson JA et al. The MIQE guidelines: minimum information for publication of quantitative real-time PCR experiments. Clin. Chem. 55(4), 611-622 (2009).

6. Lefever S, Hellemans J, Pattyn F et al. RDML: Structured language and reporting guidelines for real-time quantitative PCR data. Nucleic Acids Res. 37(7), 2065-2069 (2009).

7. Pabinger $S$, Rödiger $S$, Kriegner $A$, Vierlinger $K$, Weinhäusel A. A survey of tools for the analysis of quantitative PCR (qPCR) data. Biomol. Detect. Quantif. 1(1), 23-33 (2014).

8. Sauerwein RW, Roestenberg M, Moorthy VS. Experimental human challenge infections can accelerate clinical malaria vaccine development. Nat. Rev. Immunol. 11(1), 57-64 (2011).

9. Coffeng LE, Hermsen CC, Sauerwein RW, de Vlas SJ. The power of malaria vaccine trials using controlled human malaria infection. PLoS Comput. Biol. 13(1), e1005255 (2017).

10. Walk J, Schats R, Langenberg MCC et al. Diagnosis and treatment based on quantitative PCR after controlled human malaria infection. Malar. J. 15(1), 398 (2016).

11. King ZA, Dräger A, Ebrahim A, Sonnenschein N, Lewis $N E$, Palsson BO. Escher. a web application for building, sharing, and embedding data-rich visualizations of biological pathways. PLoS Comput. Biol. 11(8), e1004321 (2015).

12. Ince DC, Hatton L, Graham-Cumming J. The case for open computer programs. Nature. 482(7386), 485-488 (2012).

13. RDML. www.rdml.org 